\title{
Contribution of Transmission Electron Microscopy to Proton Exchange Membrane Fuel Cell Electrodes Development and Degradation Understanding
}

\author{
Laure Guétaz $^{1,2}$, Miguel Lopez-Haro, ${ }^{1,3}$, Pascale Bayle-Guillemaud ${ }^{1,3}$, Arnaud Morin ${ }^{1,2}$, Sylvie \\ Escribano $^{1,2}$ \\ 1. Univ. Grenoble Alpes, F-38000 Grenoble, France \\ 2. CEA, LITEN, F- 38054 Grenoble, France \\ 3. CEA, INAC, F-38054 Grenoble France.
}

Proton exchange membrane fuel cell (PEMFC) is one of the most promising zero emission power sources. The membrane/electrode assembly (MEA) that constitutes the fuel cell core is composed of a proton conducting membrane coated on each side with catalyst layers where the electrochemical reactions take place. These electrodes exhibit a complex porous nanostructure consisting of carbon black supporting Pt nanoparticles, ensuring electronic conduction and electro-catalysis, and of ionomer for protons transport, also used as the binder. Significant progress made on MEA components was a key factor for the recent introduction of fuel cell vehicles in the market. However a widespread commercialisation still requires reductions in costs and improvement in performance and durability. Consequently further research is needed to continue MEA component optimization and to increase understanding of their degradation mechanisms. In these research domains, transmission electron microscopy (TEM) has become an essential tool.

TEM is particularly used for analysing catalyst nanoparticles. For instance, after fuel cell operation nanoparticle coarsening was clearly observed in the cathode and the electrochemical Ostwald ripening was most often identified as the main degradation mechanism [1,2]. More recently, aberration corrected TEM coupled with electron energy loss spectroscopy (EELS) or X-Ray energy dispersive spectroscopy (EDS) have played a key role in the development of Pt alloy nanocatalysts such as Pt-Co alloys presenting higher mass-specific activity than Pt towards oxygen reduction reaction. They are the only characterization techniques able to reveal at the atomic scale the distribution of $\mathrm{Pt}$ and Co within the nanoparticles. Thereby, Pt and Co elemental maps have clearly revealed that the non-noble metal is dissolved during fuel cell operation and that a shell of pure Pt forms around the Pt-Co core [3]. These analyses have driven the developers of catalysts to synthetize new optimized Pt-Co nanoparticles with a thin Pt shell (2-3 atomic layers thick) surrounding a Pt-Co core so as to prevent the Co dissolution (Figure 1a).On the other hand, the observations in high-angle annular dark field scanning TEM (HAADF/STEM) mode of cathode Pt-Co catalysts after fuel cell operation have also revealed the presence of a large number of hollow pure Pt nanoparticles that could result from the Kirkendall effect (figure 1b) [4]. The fabrication of hollow nanoparticles has then appeared as a possible new way for reducing the amount of the costly Pt metal while keeping a large Pt surface area.

The other catalyst layer component that plays a crucial role in the fuel cell performance is the ionomer network that must ensure both the ionic contact with a maximum number of Pt nanoparticles and the connection of the ionic conduction paths to the membrane without inhibiting the gas diffusivity. However, the ionomer network is hard to be imaged because the ionomer mainly forms an ultrathin layer surrounding the carbon support that has the same contrast. Recent developments of high performance EDS detectors provide new possibilities for imaging this ionomer network. Fluorine being one of the main elements of the ionomer, ionomer distribution within the catalyst layer can now be 
visualized by acquiring fluorine EDS elemental map through thin ultramicrotomed MEA samples [5]. Hence it is now possible to assess the homogeneity of the ionomer distribution through the electrode (Figure $2 \mathrm{a}, \mathrm{b}$ ). The ionomer degradation during fuel cell operation can also be studied. In particular, the contamination of the ionomer by metallic ions coming from the degradation of alloy catalysts has been highlighted.

In parallel, the 3D morphology of the ultrathin ionomer layer surrounding the carbon particles was successfully imaged by electron tomography in HAADF-STEM mode (Figure 2 c) [6]. For this experiment, the ionomer contrast was enhanced by selectively staining the ionic domains with $\mathrm{Cs}^{+}$ions. In order to avoid high contrast of the Pt nanoparticles (compared to the ionomer contrast), a model active layer consisting of ionomer and carbon black only was made using electrode manufacturing process. The analyses reveal that doubling the amount of ionomer in the electrode leads to a twofold increase in its degree of coverage of the carbon, while the thickness of the layer, around $7 \mathrm{~nm}$, remains unchanged.

\section{References:}

[1] P. J. Ferreira et al. Electrochem. Soc. 152 -11 (2005), p. A2256.

[2] L. Guétaz, S. Escribano, O. Sicardy, J. of Power Sources, 212 (2012), p. 169.

[3] H. L. Xin et al Nano Lett. 12-1, (2011), p. 490.

[4] L. Dubau et al, Electrochim. Acta, 56 (2011), p. 10658.

[5] D. A. Cullen et al. Electrochem. Soc., 161-10 (2014), p. F1111.

[6] M. Lopez-Haro et al, Nat Commun, 5, 5229 (2014).

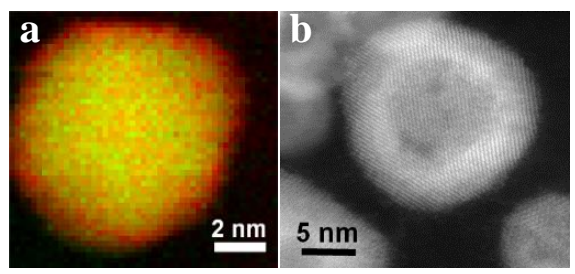

Figure 1. a) EELS Pt (red) and Co (green) elemental map of a Pt-Co nanoparticle revealing a thin Pt shell of 3 atomic layers. b) Hollow nanoparticle observed in an aged Pt-Co cathode.
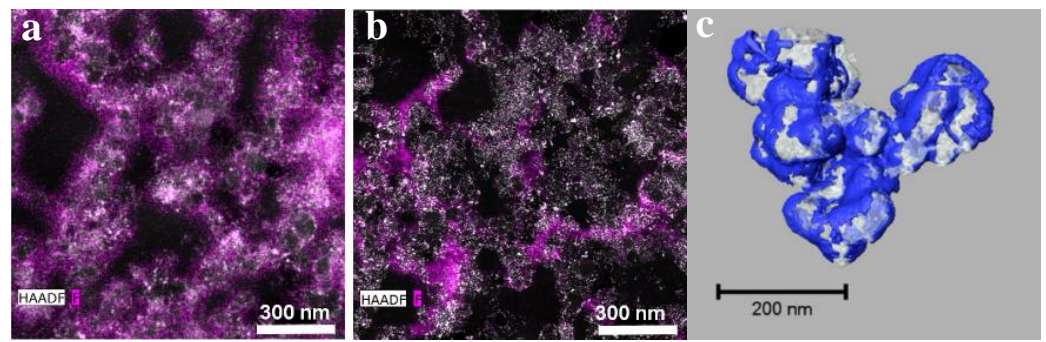

Figure 2. Fluorine (pink) EDS elemental map acquired through electrodes providing ionomer distribution image. The first electrode (a) presents a homogeneous ionomer distribution whereas the second electrode (b) shows heterogeneous ionomer distribution. (c) 3D view of the ionomer layer (in blue) surrounding the carbon black (in grey) obtained from electron tomography experiment. 